\title{
Empty convex polygons in almost convex sets*
}

\author{
Pavel Valtr \\ Department of Applied Mathematics and \\ Institute for Theoretical Computer Science (ITI), Charles University \\ Malostranské nám. 25, 11800 Praha 1, Czech Republic \\ valtr@kam.mff.cuni.cz \\ Gábor Lippner and Gyula Károlyi \\ Institute of Mathematics, Eötvös University \\ Pázmány Péter sétány 1/C, H-1117 Budapest, Hungary \\ $\{$ lipi,karolyi\}@cs.elte.hu
}

August 15, 2007

\begin{abstract}
A finite set of points, in general position in the plane, is almost convex if every triple determines a triangle with at most one point in its interior. For every $\ell \geq 3$, we determine the maximum size of an almost convex set that does not contain the vertex set of an empty convex $\ell$-gon.
\end{abstract}

\section{Introduction}

A configuration is a finite set of points in the plane such that no 3 points lie on a line. We say that a configuration is in convex position, if it is the vertex set of a convex polygon. In 1935 Erdős and Szekeres proved the following classical result:

Theorem 1 (Erdös and Szekeres [2]) For any $n \geq 3$, there is an integer $F(n)$ such that any configuration of at least $F(n)$ points contains $n$ points in convex position.

For a configuration $X$, a subset $P \subseteq X$ in convex position is called an empty polygon (in $X$ ), if the interior of the convex hull $\operatorname{conv}(P)$ of $P$ contains no point of $X$. Answering a question of Erdős [1], Horton [5] constructed arbitrarily large configurations with no empty 7-gon. Harborth [4] showed that any configuration with more than 9 points contains an

*Research by Pavel Valtr was supported by project LN00A056 of The Ministry of Education of the Czech Republic. Gyula Károlyi has been partially supported by grant T043631 of the Hungarian National Scientific Research Fund (OTKA). 
empty pentagon. The long standing open problem about the existence of empty hexagons in sufficiently large configurations was settled recently in the affirmative independently by Gerken [3] and Nicolás [8], see also Valtr [11].

We say that a configuration $X$ is $k$-convex, if the interior of every triangle determined by $X$ contains at most $k$ points of $X$. It is known that large $k$-convex configurations contain large empty polygons:

Theorem 2 (Valtr [9]) For any $k \geq 1$ and $\ell \geq 3$, there is a (smallest) integer $N(k, \ell)$ such that any $k$-convex configuration of at least $N(k, \ell)$ points contains an empty $\ell$-gon.

This result in the special case $k=1$ was first proved by Károlyi, Pach and Tóth [6], an exponential upper bound for $N(1, \ell)$ being proved later by Kun and Lippner [7]. It is known (see [7]), that $N(k, \ell)$ is at most double exponential in $k+\ell$, but it is possible that $N(k, \ell)$ is only exponential in $k+\ell$. The upper bound

$$
N(k, \ell) \leq 2^{\left(\begin{array}{l}
k+\ell \\
k+2
\end{array}\right)-1}+1
$$

was established by Valtr in [10]. Write

$$
M_{\ell}:= \begin{cases}2^{(\ell+1) / 2}-1, & \text { for } \ell \geq 3 \text { odd } \\ \frac{3}{2} 2^{\ell / 2}-1, & \text { for } \ell \geq 4 \text { even }\end{cases}
$$

The exponential lower bound $N(1, \ell) \geq M_{\ell}$ was observed and proved for odd values of $\ell$ in [6], whereas a general upper bound $N(1, \ell) \leq 2^{\lceil(2 \ell+5) / 3\rceil}-1$ is available in [7]. In this paper we determine the exact value of $N(1, \ell)$ :

Theorem 3 For any $\ell \geq 3$,

$$
N(1, \ell)=M_{\ell}
$$

\section{The lower bound construction}

For odd integers $\ell \geq 3$, a construction of a 1-convex set of $M_{\ell}-1$ points that does not contain any empty $\ell$-gon has been given in [6]. It can be easily modified to even values of $\ell$. Informally, the construction for even values of $\ell$ starts for $\ell=4$ with the non-convex configuration of four points marked by 4 in Fig. 1 . The ten points marked by 4 and 6 form the configuration for $\ell=6$, and all the 22 points shown on the figure form the configuration for $\ell=8$. Then the construction continues in a similar way for larger even values of $\ell$.

For the sake of completeness we include a more formal description following the exposition in [6]. We need the following recursive construction. Let $R_{1}$ be a set of four points in the plane such that three of them are the vertices of an equilateral triangle with the fourth point at its centre. Assume that we have already defined $R_{1}, \ldots, R_{j}$ so that

(i) $X_{j}:=R_{1} \cup \ldots \cup R_{j}$ is in general position,

(ii) the vertex set of the polygon $P_{j}:=\operatorname{conv}\left(X_{j}\right)$ is $R_{j}$, and

(iii) any triangle determined by $R_{j}$ contains precisely one point of $X_{j}$ in its interior.

Let $z_{1}, z_{2}, \ldots, z_{r}$ denote the vertices of $P_{j}$ in clockwise order, and let $\varepsilon_{j}, \delta_{j}>0$. For any $1 \leq i \leq r$, let $l_{i}$ denote the line through $z_{i}$ orthogonal to the bisector of the angle of $P_{j}$ at 
$z_{i}$. Let $z_{i}^{\prime}$ and $z_{i}^{\prime \prime}$ be two points on $l_{i}$, at distance $\varepsilon_{i}$ from $z_{i}$. Finally, move $z_{i}^{\prime}$ and $z_{i}^{\prime \prime}$ away from $P_{j}$ by a distance $\delta_{j}$, in the direction orthogonal to $l_{i}$, and denote the resulting points by $u_{i}^{\prime}$ and $u_{i}^{\prime \prime}$, respectively. It is easy to see that if $\varepsilon_{j}$ and $\delta_{j} / \varepsilon_{j}$ are sufficiently small, then $R_{j+1}:=\left\{u_{i}^{\prime}, u_{i}^{\prime \prime} \mid i=1,2, \ldots, r\right\}$ also satisfies the above three conditions.

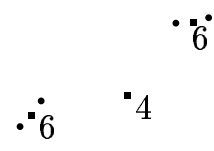

6

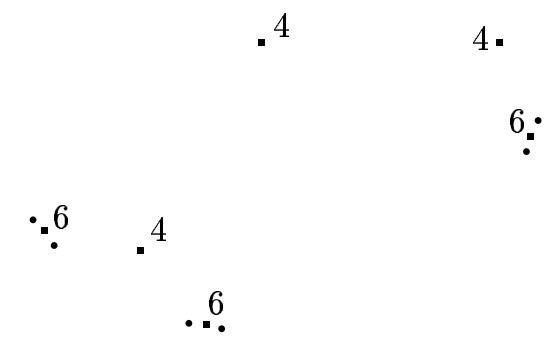

Figure 1: The construction for $\ell$ even.

We have to verify only the last condition. If $a \in\left\{u_{i}^{\prime}, u_{i}^{\prime \prime}\right\}, b \in\left\{u_{j}^{\prime}, u_{j}^{\prime \prime}\right\}$, and $c \in\left\{u_{k}^{\prime}, u_{k}^{\prime \prime}\right\}$ are three points of $R_{j+1}$, for three distinct indices $i, j, k$, then any point of $X_{j+1}=X_{j} \cup R_{j+1}$ which belongs to the interior of $\triangle a b c$ must coincide with the unique point of $X_{j}$ in the interior of $\triangle z_{i} z_{j} z_{k}$. If there exist $i \neq k$ such that $a=u_{i}^{\prime}, b=u_{i}^{\prime \prime}$, and $c \in\left\{u_{k}^{\prime}, u_{k}^{\prime \prime}\right\}$, then the only point of $X_{j+1}$ inside $\triangle a b c$ is $z_{i}$.

In view of Lemma 2.1 in [6], which claims that a configuration $X$ is 1-convex if and only if any triangle determined by three vertices of $\operatorname{conv}(X)$ contains at most one point of $X$ in its interior, $X_{n}$ is a 1-convex configuration for every positive integer $n$. Since $|X|=4$ and obviously $\left|R_{j}\right|=3 \cdot 2^{j-1}$ for $j \geq 2$, it follows that $\left|X_{n}\right|=3 \cdot 2^{n}-2$ for every positive integer $n$. Figure 1 depicts a candidate for $X_{3}$, the 12 unmarked points forming the set $R_{3}$. Since no three vertices of an empty convex polygon determined by $X_{n}$ belong to the same $R_{j}$ for $j \geq 2$, and at most three vertices can belong to $R_{1}$, it follows that any such polygon has at most $2 n+1$ vertices.

With $\ell=2 n+2$ we have constructed a 1 -convex configuration $X_{n}$ of $M_{\ell}-1$ points that does not contain an empty $\ell$-gon. The inequality $N(1, \ell) \geq M_{\ell}$ is thus fully established.

\section{Proof of the upper bound}

It remains to show that any 1-convex configuration of size at least $M_{\ell}$ contains an empty $\ell$-gon. For a set $Y \subseteq X$, let $i(Y)$ be the number of points of $X$ lying in the interior of $\operatorname{conv}(Y)$. An $\langle r, s\rangle$-configuration is a 1-convex configuration $X$ containing a subset $Y \subseteq X$ of size $r+s$ such that $X \cap \operatorname{conv}(Y)=Y$ and $i(Y) \leq s$. 
Here is our key lemma which shows, roughly speaking, that any configuration with (at most) $s \geq 3$ interior points contains a large subconfiguration with at most $s-1$ interior points:

Lemma 1 If $r \geq s+3 \geq 6$, then any $\langle r, s\rangle$-configuration is an $\langle r+2-t, s-1\rangle$-configuration, where $t:=\lceil(r+1) /(s+2)\rceil \geq 2$.

Proof. Let $r \geq s+3 \geq 6$ and let $X$ be an $\langle r, s\rangle$-configuration. We want to show that $X$ is an $\langle r+2-t, s-1\rangle$-configuration. Let $Y \subseteq X$ be a subset witnessing that $X$ is an $\langle r, s\rangle$-configuration.

If $i(Y)<s$, then the removal of $t-1$ rightmost points from $Y$ gives a set $Y^{\prime} \subset Y$ of size $(r+2-t)+(s-1)$ such that $i\left(Y^{\prime}\right) \leq s-1$. Thus, we may suppose that $i(Y)=s$. Then $X$ contains the vertices of a convex $r$-gon $P=p_{0} p_{1} \ldots p_{r-1}$ containing exactly $s$ points of $X$ in the interior.

Let $u$ denote the smallest integer such that $r+1-u t \leq 2 t$. Since $t \geq(r+1) /(s+2)$, we have $r+1-s t \leq 2 t$ and thus $u \leq s$. From $r+1-t>2 t$ it also follows that $u \geq 2$. Consequently, we can partition the interior of $P$ into the following $u+2$ convex polygons (see Fig. 2):

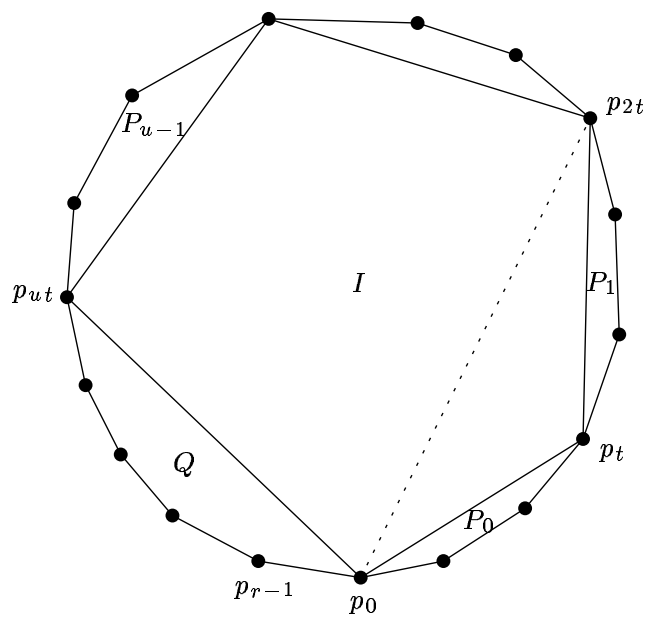

Figure 2: The partition of the interior of $P$ (case $r=17, s=5)$.

- the $(u+1)$-gon $I=p_{0} p_{t} p_{2 t} \ldots p_{u t}$,

- the $(t+1)$-gons $P_{i}=p_{i t} p_{i t+1} \ldots p_{(i+1) t}(i=0,1, \ldots, u-1)$,

- the polygon $Q=p_{u t} p_{u t+1} \ldots p_{r-1} p_{0}$.

If a polygon $P_{i}$ is not empty for some $0 \leq i \leq u-1$, then the convex hull of the set

$$
(P \cap X) \backslash\left\{p_{i t+1}, p_{i t+2}, \ldots, p_{(i+1) t-1}\right\}
$$

contains $r+s-(t-1)=(r+2-t)+(s-1)$ points of $X$ in total and at most $s-1$ points of $X$ in the interior. Thus, $X$ is an $\langle r+2-t, s-1\rangle$-configuration in this case. So we may 
suppose that the polygons $P_{0}, \ldots, P_{u-1}$ are empty. Since $X$ is 1 -convex and the $(u+1)$-gon $I$ can be triangulated into $u-1$ triangles, the interior of $I$ contains at most $u-1 \leq s-1$ points. It follows that the polygon $Q$ is not empty. Note that $Q$ has $r+1-u t \leq 2 t$ vertices.

The above argument does not depend on the particular choice of the vertex $p_{0}$ of $P$. Thus we may assume that the $2 t$-gons $Q_{i}=p_{i} p_{i+1} \ldots p_{i+(2 t-1)}$ are not empty for $i=0, \ldots, 2 t-1$. Let $q \in X$ lie inside $Q_{0}$. Since $4 t-2 \leq r$, the polygons $Q_{0}, Q_{2 t-1}$ have disjoint interiors, and there is a $j \in\{0,1, \ldots, 2 t-2\}$ with $q \in Q_{j}, q \notin Q_{j+1}$. Without loss of generality, we may assume that $j=0$. Since $Q_{1}$ is not empty and $q \notin Q_{1}$, there is a point $q^{\prime} \in X, q^{\prime} \neq q$, lying inside $Q_{1} . Q_{0} \cup Q_{1}$ is covered by the polygons $P_{0}, P_{1}$ and by the triangle $T=p_{0} p_{t} p_{2 t}$. Since $X$ is 1 -convex, it follows that one of the polygons $P_{0}, P_{1}$ is not empty. As we have already shown above, this implies that $X$ is an $\langle r+2-t, s-1\rangle$-configuration.

Corollary 1 (i) If $\ell \geq 5$ is odd and $1 \leq j \leq(\ell-3) / 2$, then any $\left\langle(\ell-2 j-1) 2^{j}+1,2^{j+1}-2\right\rangle$ configuration is a $\langle 2 \ell-5,2\rangle$-configuration.

(ii) If $\ell \geq 4$ is even and $1 \leq j \leq(\ell-2) / 2$, then any $\left\langle 3(\ell-2 j) 2^{j-1}+1,3 \cdot 2^{j}-2\right\rangle$ configuration is a $\langle 2 \ell-5,2\rangle$-configuration.

Proof. (i) For the case $j=1$, there is nothing to prove. We further continue by induction on $j$. If $j>1$, then repeated applications of Lemma 1 show that any $\left\langle(\ell-2 j-1) 2^{j}+1,2^{j+1}-2\right\rangle$ configuration is an $\langle r, s\rangle$-configuration for the pairs

$$
\begin{aligned}
\langle r, s\rangle= & \left\langle(\ell-2 j-1) 2^{j}+1-1 \cdot \frac{\ell-2 j-3}{2}, 2^{j+1}-2-1\right\rangle, \\
& \left\langle(\ell-2 j-1) 2^{j}+1-2 \cdot \frac{\ell-2 j-3}{2}, 2^{j+1}-2-2\right\rangle, \\
& \vdots \\
& \left\langle(\ell-2 j-1) 2^{j}+1-2^{j} \cdot \frac{\ell-2 j-3}{2}, 2^{j+1}-2-2^{j}\right\rangle .
\end{aligned}
$$

Accordingly, it is an $\left\langle(\ell-2(j-1)-1) 2^{j-1}+1,2^{j}-2\right\rangle$-configuration, thus by the inductive hypothesis it is also a $\langle 2 \ell-5,2\rangle$-configuration.

(ii) If $j=1$, then two applications of Lemma 1 show that any $\langle 3 \ell-5,4\rangle$-configuration is a $\langle 5 \ell / 2-5,3\rangle$-configuration, and further also a $\langle 2 \ell-5,2\rangle$-configuration. We continue by induction on $j$. If $j>1$, then once again a repeated application of Lemma 1 implies that any $\left\langle 3(\ell-2 j) 2^{j-1}+1,3 \cdot 2^{j}-2\right\rangle$-configuration is an $\langle r, s\rangle$-configuration for the pairs

$$
\begin{aligned}
\langle r, s\rangle= & \left\langle 3(\ell-2 j) 2^{j-1}+1-1 \cdot \frac{\ell-2 j-2}{2}, 3 \cdot 2^{j}-2-1\right\rangle, \\
& \left\langle 3(\ell-2 j) 2^{j-1}+1-2 \cdot \frac{\ell-2 j-2}{2}, 3 \cdot 2^{j}-2-2\right\rangle, \\
& \vdots \\
& \left\langle 3(\ell-2 j) 2^{j-1}+1-3 \cdot 2^{j-1} \cdot \frac{\ell-2 j-2}{2}, 3 \cdot 2^{j}-2-3 \cdot 2^{j-1}\right\rangle .
\end{aligned}
$$

Accordingly, it is a $\left\langle 3(\ell-2(j-1)) 2^{j-2}+1,3 \cdot 2^{j-1}-2\right\rangle$-configuration, thus by the inductive hypothesis it is also a $\langle 2 \ell-5,2\rangle$-configuration. 
We are ready to finish the proof of Theorem 3 . We still need to prove that any 1 -convex configuration $X$ of size $M_{\ell}$ contains an empty $\ell$-gon. The case $\ell=3$ is trivial. Thus, let $\ell \geq 4$.

The convex hull of $X$ has at least $\left\lceil\left(M_{\ell}+2\right) / 2\right\rceil=\left(M_{\ell}+3\right) / 2$ vertices, since otherwise any triangulation of $\operatorname{conv}(X)$ contains a triangle with more than one point in the interior. Thus, $X$ itself witnesses that it is an $\left.\left\langle M_{\ell}+3\right) / 2,\left(M_{\ell}-3\right) / 2\right\rangle$-configuration. Now, Corollary 1 for $j=(\ell-3) / 2$ (if $\ell$ is odd) or for $j=(\ell-2) / 2$ (if $\ell$ is even) shows that $X$ is a $\langle 2 \ell-5,2\rangle$ configuration.

Let $Y \subseteq X$ witness that $X$ is a $\langle 2 \ell-5,2\rangle$-configuration. If $i(Y)=0$, then $Y$ is an empty $(2 \ell-3)$-gon and the statement follows from $2 \ell-3 \geq \ell$. Now, let $i(Y)>0$, and let $y_{1} \in Y$ be an interior point of $\operatorname{conv}(Y)$. If $i(Y)=2$, then we choose $y_{2} \in Y$ as the other interior point of $\operatorname{conv}(Y)$. Otherwise $i(Y)=1$ and we fix any point $y_{2} \in Y \backslash\left\{y_{1}\right\}$. By the pigeonhole principle, there are at most $\ell-3$ points of $Y \backslash\left\{y_{1}, y_{2}\right\}$ on one of the sides of the line $y_{1} y_{2}$. We remove these points from $Y$. The remaining points of $Y$ form an empty polygon with at least $\ell$ vertices. Theorem 3 is proved.

\section{References}

[1] P. Erdős, On some problems of elementary and combinatorial geometry, Ann. Mat. Pura. Appl. (4) 103 (1975), 99-108.

[2] P. Erdős and Gy. Szekeres, A combinatorial problem in geometry, Compositio Math. 2 (1935), 464-470.

[3] T. Gerken, Empty convex hexagons in planar point sets, Discrete Comput. Geom., to appear.

[4] H. Harborth, Konvexe Fünfecke in ebenen Punktmengen, Elem. Math. 33 (1978), 116118.

[5] J. D. Horton, Sets with no empty convex 7-gons, Canadian Math. Bull. 26 (1983), 482484 .

[6] Gy. Károlyi, J. Pach, and G. Tóth, A modular version of the Erdős-Szekeres theorem, Studia Sci. Math. Hungar. 38 (2001), 245-259.

[7] G. Kun and G. Lippner, Large convex empty polygons in $k$-convex sets, Period. Math. Hungar. 46 (2003), 81-88.

[8] C. Nicolás, The empty hexagon theorem, Discrete Comput. Geom., to appear.

[9] P. Valtr, A sufficient condition for the existence of large empty convex polygons, Discrete Comput. Geom. 28 (2002), 671-682.

[10] P. Valtr, Open caps and cups in planar point sets, Discrete Comput. Geom. 37 (2007), $565-576$.

[11] P. Valtr, On the empty hexagons theorem, submitted. 